\title{
A Circular Split Ringdouble Negative Metamaterial Having Simultaneous Negative Permittivity and Permeability
}

\author{
Muhammad Waqas ${ }^{1}$, Muhammad Abid Saeed ${ }^{1}$, Muhammad Junaid khan ${ }^{2}$, \\ Muhammad Tayyab Qaiser ${ }^{3}$ \\ ${ }^{I}$ Department of Electrical Engineering, Sarhad University of Science and IT, Peshawar, Pakistan \\ ${ }^{2}$ Department of Communications Engineering, University of Oulu, Finland \\ ${ }^{3}$ COMSATS University, Abbottabad, Pakistan
}

\begin{abstract}
The scope of this paper is to design and simulate a novel circular split ring structure having simultaneous negative permittivity and permeability so called double negative metamaterial or left handed material. The DNG structure consists of five circular split ring resonators on one side of dielectric medium and a couple of circular wires on other side. The complex permittivity, permeability, impedance and refractive index are determined from simulated Scattering parameters using direct retrieval method. Simulations of DNG structure are carried out using CST MWS. MATLAB is used for verification of negative values of structure's parameter.
\end{abstract}

Keywords:Double negative left handed material, Negative Permittivity, Negative Permeability, Negative Refractive Index, Negative impedance, Split Ring Resonators.

\section{Introduction}

Metamaterials are artificial material having numerous potential applications in science, technology and medicine which have been receiving increased attention recently. These novel artificial materials are first predicted by V. Veselago but the experimental verification did not occur until several decades [1]. Later several researchers studied the characteristics and applications of SRR based left handed material (LHM) for experimental verification. Double negative materials do not exist in nature but such material is physically realizable by making composite structure in which the propagation of electromagnetic waves are possible [2]. To achieve negative permittivity and permeability, circular split ring resonatorsare used on one side of a dielectric medium while a couple of circular wires on other side. These SRR gives negative permittivity and wires gives negative permeability. Furthermore negative refractive index and impedance are also achieved from respective structure. The DNG structure has been simulated in CST microwave studio 2011 software and scattering parameters are extracted to calculate negative values of permittivity, permeability, impedance and refractive index using direct retrieval method [3-5].

The DNG materials have a number of applications in microwave engineering [6]. For metamaterials, it is implied that are concerned in their response to electromagnetic fields. The most frequently application of DNG is to use it as a substrate or superstrate for antenna to improve its directivity and gain [7-9]. Other applications such as metamaterials-cloaking is one of the magical application of metamaterial. Perfect lenses and near field imaging is also the attractive ideas of metamaterial [6].

\section{Methodology}

For double negative structure using CST Microwave studio five circular split rings of copper having different outer and inner radius are used on dielectric medium (substrate) and five circularwires of same length and radius are used on other side of dielectric medium. The substrate used here is FR4 having a dielectric of 4.3 and the thickness of substrate is $1 \mathrm{~mm}$. The structure of DNG front view (Circular SRR) and back view (wires) are shown in Figure.1 and Figure. 2 respectively. For convenience, parameters of circular split ring, wires and substrate are summarized respectively in Table I.

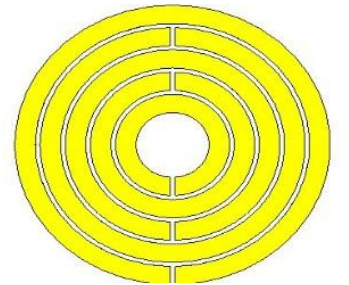

Figure.1: Circular SRR (Front View) 


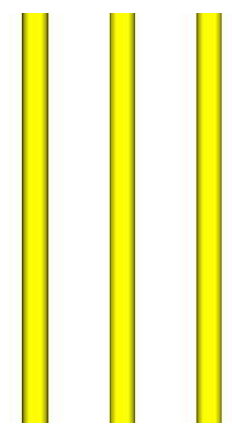

Figure.2: Wires (back view)

Table I: Parameters of DNG Structure (All dimensions are in $\mathrm{mm}$ )

\begin{tabular}{|c|c|}
\hline Parameters of DNG & Values \\
\hline Length of each wire & 14.2 \\
\hline Radius of each wire & 0.45 \\
\hline Inner and outer radius of ring 1 & $2.5 \& 1.6$ \\
\hline Inner and outer radius of ring 2 & $3.6 \& 2.7$ \\
\hline Inner and outer radius of ring 3 & $4.7 \& 3.8$ \\
\hline Inner and outer radius of ring 4 & $5.8 \& 4.9$ \\
\hline Inner and outer radius of ring 5 & $6.9 \& 6.0$ \\
\hline Dielectric of substrate & 4.3 \\
\hline Thickness of substrate (FR4) & 1 \\
\hline Gap of each rings & 0.12 \\
\hline
\end{tabular}

Several techniques have been used to get negative permittivity and permeability from the simulated complex value of S-parameter. Nicolson Ross Weir equations is one of the most repeated method to find negative values of permittivity and permeability [10]-[13]. Another method used Drude model and Lorentz model made some assumptions about negative permittivity and permeability [2], [14]. Among these and all other methods a direct retrieval method is used in this work which is more convenient and simple [3].

The suggested DNG Metamaterial model is configuring between two waveguide ports at the left and right of Y-Axis. The wave is excited from positive Y-axis (Port 1) towards negative Y-axis (Port 2) to calculate complex S11 (Transmission/reflection of port 1 to 1) and complex S21 (Transmission/ Reflection of port 1 to 2) parameters. The X-Plane is defined as a Perfect Electric Boundary (PEB) while Z-plane is defined as a Perfect Magnetic boundary (PMB). Simulated DNG structure between two waveguide ports is shown in Figure.3.

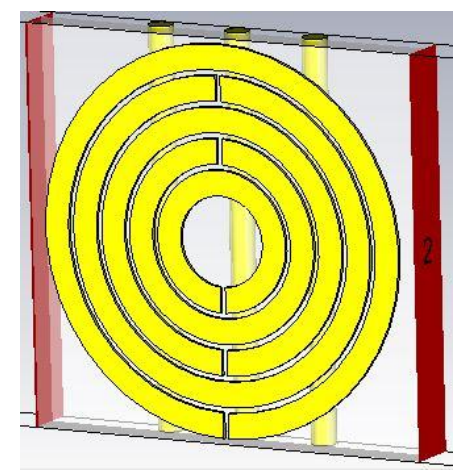

Figure.3: DNG structure between two waveguide ports

Transmission/Reflection method is used in order to obtain S-parameters from corresponding structure. The real and imaginary values of scattering parameters are then exported to MATLAB to obtained negative values using permittivity and permeability equations.

Permittivity and permeability of a material are given by [3] :

and

$$
\varepsilon=n / z
$$

$\mu=n z(2)$ 
where,

$n=\frac{1}{k d} \cos ^{-1}\left[\frac{1}{2 S_{21}}\left(1-S_{11}^{2}+S_{21}^{2}\right)\right]$

and

$z=\sqrt{\frac{\left(1+S_{11}\right)^{2}-S_{21}^{2}}{\left(1-S_{11}\right)^{2}-S_{21}^{2}}}$

\section{Simulated Results and Discussion}

Scattering parameter is the key term for extracting negative permittivity and permeability. The complex scattering parameters S11, S12, S21 \& S22 are extracted from CST Microwave studio. The structure resonateswithin the given range of frequency as shown in the following Figures. Figure.4 and Figure.5 show the reflection graphs and Figure.6 and Figure.7 shows the transmission graphs.

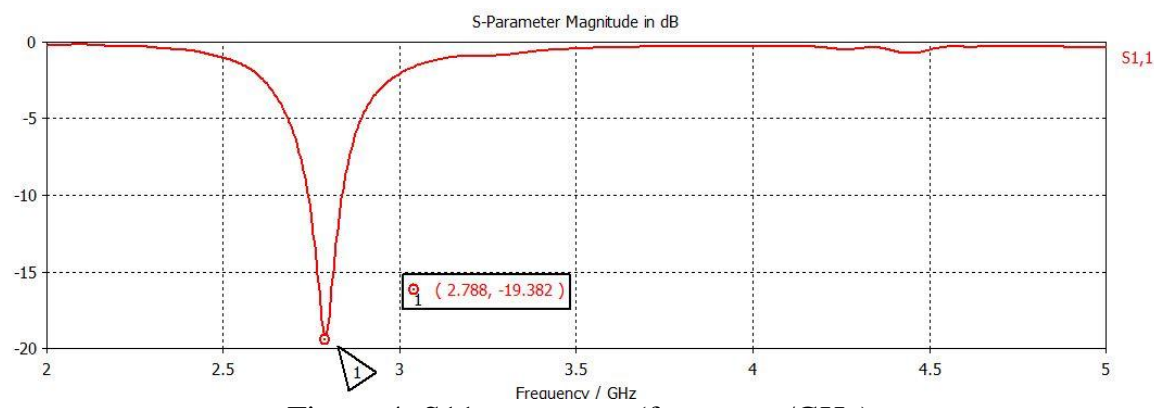

Figure.4: S11-parameter (frequency/GHz)

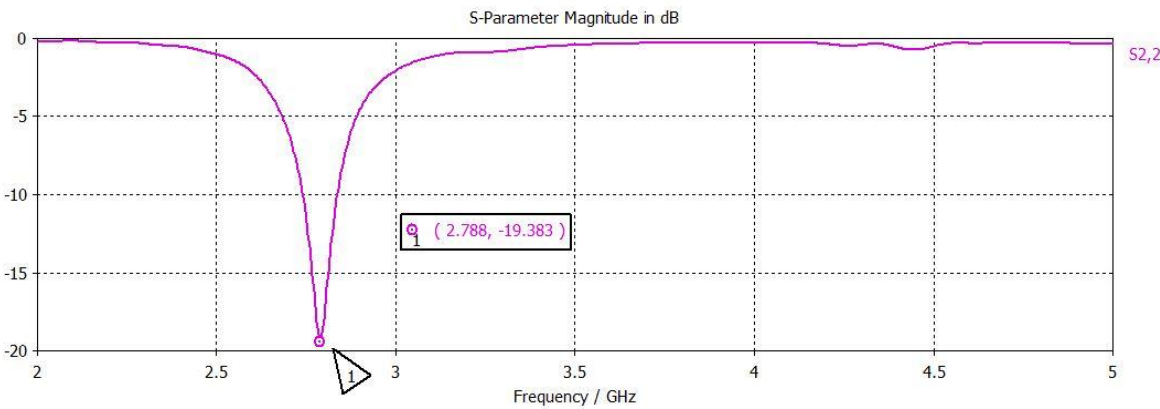

Figure.5: S22-parameter (frequency/GHz)

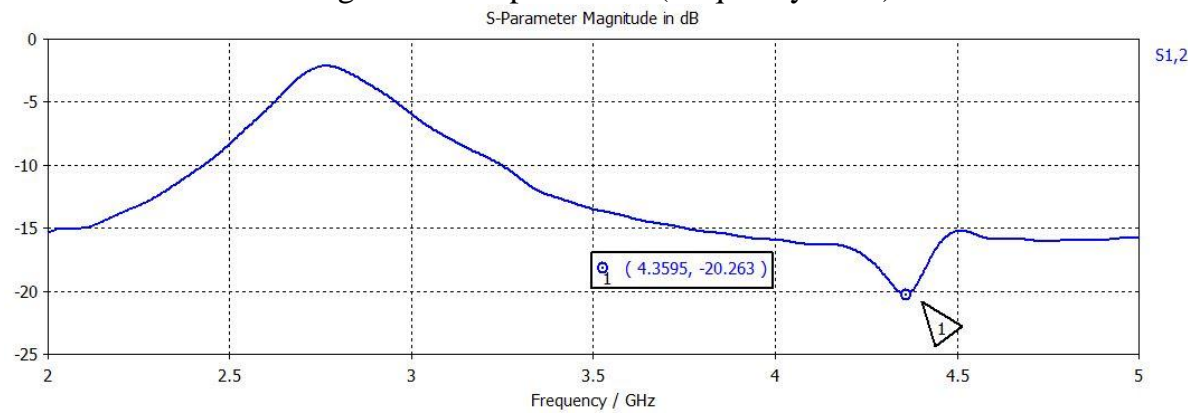

Figure.6: S12-parameter (frequency/GHz)

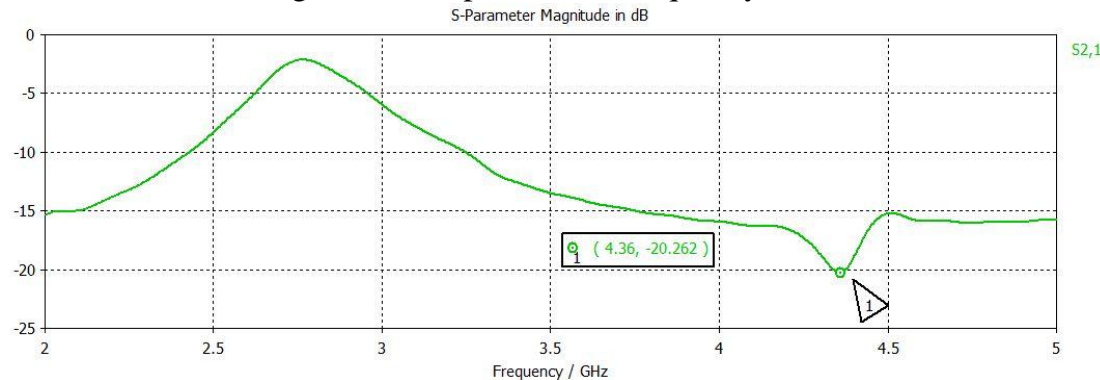

Figure.7: S21-parameter (frequency/GHz) 
The complex values of these parameters are than exported to MATLAB and using previous equations of direct retrieval method, the graphs of negative permittivity and permeability versus frequency are obtained. These graphs provided verified results of a negative behavior of such periodic structure. It confirmed that proposed structure is double negative material. Negative permittivity and permeability graphs are shown in Figure. 8 and Figure. 9 respectively.

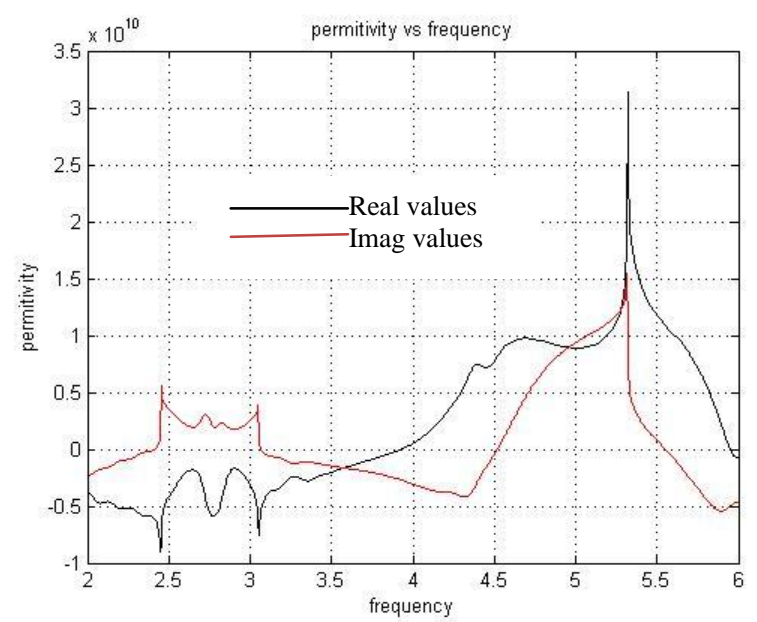

Figure.8: Proposed DNG permittivity vs frequency

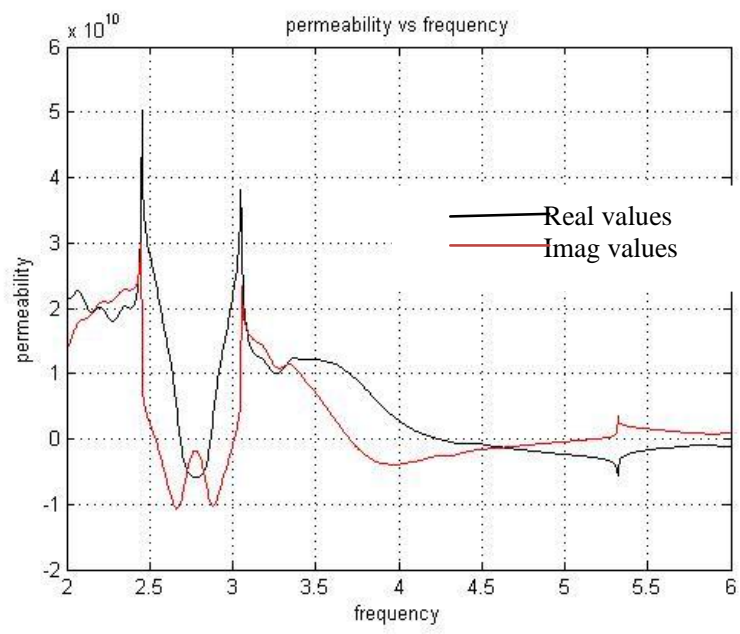

Figure.9: Negative permeability vs frequency

Negative refracitve index and impedance are also found from the MATLAB software using the same direct retrieval method as shown in Figure.10 and Figure.11. These graphs showed negative values of refractive index and impedance in the given frequency range.

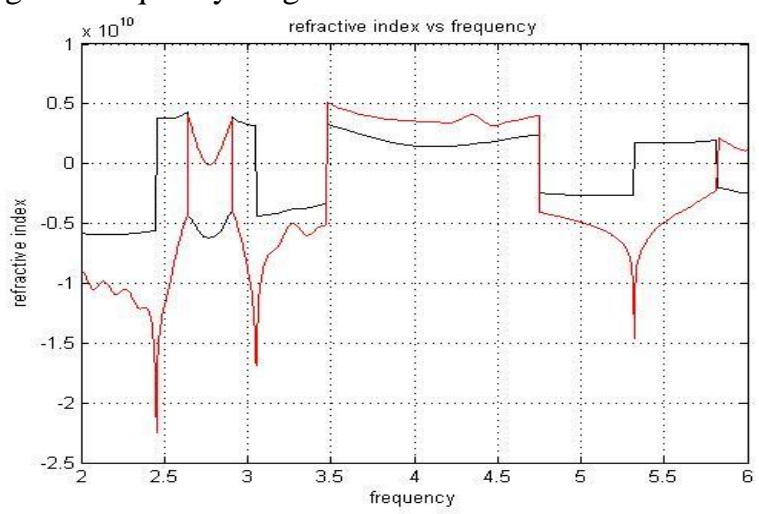

Figure.10: Negative Refracti ${ }^{\ldots} \ldots$ Real values Imag values 


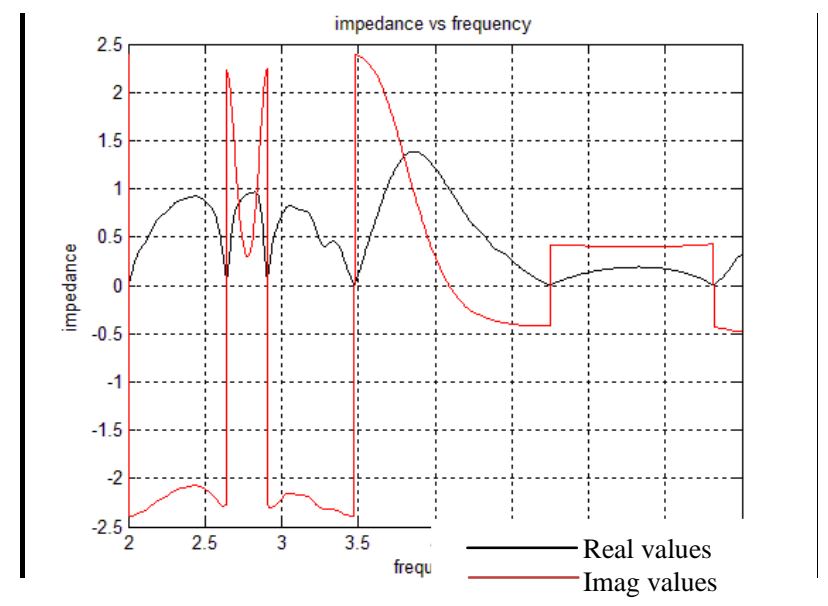

Figure.11: Negative Impedance vs frequency

\section{Conclusion}

All the simulations are successfully done using CST MWS while the negative values of refractive index, imepedance, permittivity and permeability are fruitfully obtained using MATLAB from transmission reflection method using direct retrieval technique. Negative paremters are obtained at different operating frequency which is the finestoption for the selection of respective DNG structure. The simulated structure possesses simultenous negative permittivity and permeability at different frequency range having left handed properties and hence called LHM or DNG. Further amendments in this structure can give favorite values of constitutive parameters for desired frequency ranges. DNG metamaterial structures can be of great importance where negative refractive index, enhanced gain and radiation characteristics are need at specific range of frequency. The structure is widely used for the improvement of diverse antenna's parameters.

\section{Refernces}

[1] V. G. Veselago, "The Electrodynamics of Substances with Simultaneously Negative values of $\varepsilon$ and $\mu$," Soviet Phys Usp 10 (1968), 509-514.

[2] M. F. Khan, "Tunable Metamaterials," PhD. thesis, Ghulam Ishaq Khan Institute of Engineering Sciences and Technology, Topi, Swabi, Khyber Pakhtunkhwa, Pakistan. May 2011.

[3] D. R. Smith, D. C. Vier, and C. M. Soukoulis, "Electromagnetic parameter retrieval from inhomogeneous metamaterials," The American Physical Society, pp. 1-11, March 22, 2005.

[4] H. Zhou, G. Lu, Y. Li, S. Wang, and Y. Wang, "An Improved Method of Determining Permittivity and Permeability by S Parameters," PIERS processdings, beijing, chaina, vol. 11, no. 1, pp. 768-773, 2009.

[5] A. Mallik, S. Kundu, and O. Goni, "Design of a novel Two-circular U-Shaped Double Negative Metamaterial," IEEE no.978-14799-0400-6/2013.

[6] M. Lapine and S. Tretyakov, “Contemporary notes on metamaterials”,IET Microwave Antenna propag., 1, pp.3-11, 2007.

[7] H. Attia, L. Yousefi, M. M. Bait-suwailam, M. S. Boybay, and O. M. Ramahi, "Enhanced gain microstrip antenna using engineered magnetic superstrates," IEEE Antennas and Wireless Prog. Lett. vol. 8, pp. 1198-1201, 2009.

[8] D. Jin, B. Li, and J. Hong, "Gain improvement of a microstrip patch antenna using metamaterial superstrate with the zero refractive index," IEEE Trans., pp. 10-12, 2012.

[9] P. K. Singhal and B. Garg, "Design and characterization of compact microstrip patch antenna using split ring shaped metamaterial structure," Int. Journal of Electrical and Computer Engineering, vol. 2, no. 5, pp. 655-662, 2012.

[10] A. N. Vicente, G. M. Dip, and C. Junqueira, "The step by step development of NRW method," 2011 SBMO/IEEE MTT-S Int. Microw. Optoelectron. Conf. (IMOC 2011), pp. 738-742, Oct. 2011.

[11] A. L. de Paula, M. C. Rezende, and J. J. Barroso, "Modified Nicolson-Ross-Weir (NRW) method to retrieve the constitutive parameters of low-loss materials," 2011 SBMO/IEEE MTT-S Int. Microw. Optoelectron. Conf. (IMOC 2011), pp. 488-492, Oct. 2011.

[12] O. Luukkonen, S. I. Maslovski, and S. A. Tretyakov, “A Stepwise Nicolson-Ross-Weir-Based Material Parameter Extraction Method," IEEE Antennas and Wireless Propagation Letters vol. 10, pp. 1295-1298, 2011.

[13] R. W. Ziolkowski, "Design, fabrication, and testing of double negative metamaterials," IEEE Trans. Antennas Propag., vol. 51, no. 7, pp. 1516-1529, Jul. 2003.

[14] J. Franklin, J. Biddle, Bohdan Balko, "Double Negative Materials ( DNM ) Phenomena and Applications," Institute of Defense Analysis central research Program, July 2009. 